\title{
9 Referenzen
}

Adriaenssens N, Coenen S, Versporten A, Muller A, Minalu G, Faes C, Vankerckhoven V, Aerts M, Hens N, Molenberghs G, Goossens H on behalf of the ESAC Project Group (2011) European Surveillance of Antimicrobial Consumption (ESAC): Outpatient antibiotic use in Europe and association with resistance: a cross-national database study (1997-2009). J Antimicrob Chemother 66(suppl 6):vi3-v12.

Albrich WC, Monnet DL, Harbarth S (2004): Antibiotic selection pressure and resistance in Streptococcus pneumoniae and Streptococcus pyogenes. Emerging Infectious Diseases 10(3), 514-517. http://wwwnc.cdc.gov/eid/ article/10/3/03-0252.htm (abgerufen am 28. August 2012).

Alteri CJ \& Mobley HL (2012): Escherichia coli physiology and metabolism dictates adaptation to diverse host microenvironments. Curr Opin Microbiol 15:3-9.

Allison KR, Brynildsen MP, Collins JJ (2011): Metabolite-enabled eradication of bacterial persisters by aminoglycosides. Nature 473:216-220.

Aminov RI (2009): The role of antibiotics and antibiotic resistance in nature. Environ Microbiol 11:2970-2988.

Amsden GW (2005): Anti-inflammatory effects of macrolides - an underappreciated benefit in the treatment of community-acquired respiratory tract infections and chronic inflammatory pulmonary conditions? J Antimicrob Chemother 55:10-21.

Arias CA, Mendes RE, Stilwell MG, Jones RN, Murray BE (2012): Unmet Needs and Prospects for Oritavancin in the Management of Vancomycin-Resistant Enterococcal Infections. Clinical Infectious Diseases 54(suppl 3):233-238. DOI:10.1093/cid/cir924.

Baquero F, Coque TM, de la Cruz F (2011): Ecology and evolution as targets: the need for novel eco-evo drugs and strategies to fight antibiotic resistance. Antimicrob Agents Chemother 55:3649-3660.

Blaser M (2011): Stop the killing of beneficial bacteria. Nature 476:393-394.

Boston Business Journal (2012): Cubist: Cubicin revenues grew to \$735M in 2011. http://www.bizjournals.com/boston/news/2012/01/09/cubist-cubicin-revenuesgrew-to-735m.html, Stand: 08. Juni 2012.

Brinster S, Lamberet G, Staels B, Trieu-Cuot P, Gruss A, Poyart C (2009): Type II fatty acid synthesis is not a suitable antibiotic target for Gram-positive pathogens. Nature 458:83-86.

Brown SA, Palmer KL, Whiteley M (2008): Revisiting the host as a growth medium. Nat Rev Microbiol 6:657-666.

Brook I \& Gillmore JD (1993): In-vitro susceptibility and in-vivo efficacy of antimicrobials in the treatment of intraabdominal sepsis in mice. J Antimicrob Chemother 31:393-401.

Bruce J, MacKenzie FM, Cookson B, Mollison J, van der Meer JW, Krcmery V, Gould IM (2009): Antibiotic stewardship and consumption: findings from a pan-European hospital study. ARPAC Steering Group. J Antimicrob Chemother. 64:853-860.

Buffie CG, Jarchum I, Equinda M, Lipuma L, Gobourne A, Viale A, Ubeda C, Xavier J, Pamer EG (2012): Profound alterations of intestinal microbiota following a single dose of clindamycin results in sustained susceptibility to Clostridium difficileinduced colitis. Infect Immun 80:62-73.

BVL - Bundesamt für Verbraucherschutz und Lebensmittelsicherheit, Paul-EhrlichGesellschaft für Chemotherapie und Zentrum Infektiologie der Universitätsklinik Freiburg (Hrsg.) (2011): GERMAP 2010: Antibiotika-Resistenz und -Verbrauch. 
Bericht über den Antibiotikaverbrauch und die Verbreitung von Antibiotikaresistenzen in der Human- und Veterinärmedizin in Deutschland. Antiinfectives Intelligence, Rheinbach.

Bundestierärztekammer (2010): Leitlinien für den sorgfältigen Umgang mit antibakteriell wirksamen Tierarzneimitteln. Beilage zum Deutschen Tierärzteblatt 10.

Butler MS \& Cooper MA (2011): Antibiotics in the clinical pipeline. J Antibiot 64:413-425.

Bywater RJ, Casewell MW (2000): An assessment of the impact of antibiotic resistance in different bacterial species and of the contribution of animal source to resistance in human infections. J Antimicrob Chemother 46(4):643-645.

Cegelski L, Marshall GR, Eldridge GR, Hultgren SJ (2008): The biology and future prospects of antivirulence therapies. Nat Rev Microbiol. 6:17-27.

Costello EK, Lauber CL, Hamady M, Fierer N, Gordon JI, Knight R (2009): Bacterial community variation in human body habitats across space and time. Science 326(5960):1694-7.

Cuny C, Friedrich A, Kozytska S, Layer F, Nübel U, Ohlsen K, Strommenger B, Walther B, Wieler L, Witte W (2010): Emergence of methicillin-resistant Staphylococcus aureus (MRSA) in different animal species. International Journal of Medical Microbiology 300 (2-3) (Februar):109-117. DOI:10.1016/j.ijmm.2009.11.002.

Dalhoff A, Ambrose PG, Mouton JW (2009): A long journey from minimum inhibitory concentration testing to clinically predictive breakpoints: deterministic and probabilistic approaches in deriving breakpoints. Infection 37:296-305.

Dalhoff A \& Shalit I (2003): Immunomodulatory effects of quinolones. Lancet Infect Dis 3:359-371.

Davies J, Spiegelman GB, Yim G (2006): The world of subinhibitory antibiotic concentrations. Curr Opin Microbiol 9:445-453.

de Kraker MEA, Davey PG, Grundmann H, on behalf of the BURDEN study group (2011): Mortality and Hospital Stay Associated with Resistant Staphylococcus aureus and Escherichia coli Bacteremia: Estimating the Burden of Antibiotic Resistance in Europe. PLoS Med 8(10):e1001104. DOI:10.1371/journal.pmed.1001104.

Dubreuil L \& Odou MF (2010): Anaerobic bacteria and antibiotics: What kind of unexpected resistance could I find in my laboratory tomorrow? Anaerobe $16: 555-559$.

EASAC (2005): Infectious diseases - importance of co-ordinated activity in Europe. London: The Royal Society. Verfügbar unter http://www.easac.eu/home/reportsand-statements/detail-view/article/infectious-d.html.

EASAC (2006): Vaccines: innovation and human health. London: The Royal Society. Verfügbar unter http://www.easac.eu/home/reports-and-statements/ detail-view/article/vaccines-in.html.

EASAC (2007): Tackling antibacterial resistance in Europe. The Royal Society. Verfügbar unter http://www.easac.eu/home/reports-and-statements/detail-view/article/ tackling-ant.html.

ECDC/EMEA (2009): The bacterial challenge: time to react. Joint technical report. Verfügbar unter http://www.ema.europa.eu/docs/en_GB/document_library/ Report/2009/11/WC50000877.pdf.

ECDC (2011): Surveillance Report. Antimicrobial resistance surveillance in Europe. Verfügbar unter: http://ecdc.europa.eu/en/publications/Publications/1111_SUR_ AMR_data.pdf (abgerufen am: 23. November 2011). 
EU-KOM (2011): Mitteilung der Europäischen Kommission an das Europäische Parlament und den Rat: Aktionsplan zur Abwehr der steigenden Gefahr der Antibiotikaresistenz. COM (2011) 748. http://ec.europa.eu/dgs/health_ consumer/docs/communication_amr_2011_748_de.pdf (abgerufen am: 28. August 2012).

Fabbretti A, Gualerzi CO, Brandi L (2011): How to cope with the quest for new antibiotics. FEBS Letters 585:1673-1681.

Fajardo A \& Martínez JL (2008): Antibiotics as signals that trigger specific bacterial responses. Curr Opin Microbiol 11:161-167.

Fears R, van der Meer J, ter Meulen V (2011): The Changing Burden of Infectious Disease in Europe. Science Translational Medicine 103(3):103cm30.

Fischbach MA \& Walsh CT (2009): Antibiotics for emerging pathogens. Science 325:1089-1093.

Gastmeier P, Rellhauer M, Heesemann J, Kern W (2009): Die Antibiotika-Resistenzstrategie: Verbesserung der Aus-, Weiter- und Fortbildung in Krankenhäusern. Klinikarzt 38:562-565.

Ginsburg I (2002): The role of bacteriolysis in the pathophysiology of inflammation, infection and post-infectious sequelae. APMIS 110:753-770.

Griffin MO, Ceballos G, Villarreal FJ (2011): Tetracycline compounds with non-antimicrobial organ protective properties: possible mechanisms of action. Pharmacol Res 63:102-107.

Gullberg E, Cao S, Berg OG, Ilbäck C, Sandegren L, Hughes D, Andersson DI (2011): Selection of resistant bacteria at very low antibiotic concentrations. PLoS Pathog 7:e1002158.

Hacker J \& Heesemann J (Hrsg.) (2002): Molecular Infection Biology: interactions between microorganisms and cells. Wiley-Spektrum, Hoboken (NJ), Heidelberg.

Hamad B (2010): The antibiotics market. Nature Rev Drug Discov 9:675-676.

Hancock RE \& Patrzykat A (2002): Clinical development of cationic antimicrobial peptides: from natural to novel antibiotics. Curr Drug Targets Infect Disord 2:79-83.

Harris SR, Feil EJ, Holden MT, Quail MA, Nickerson EK, Chantratita N, Gardete S, Tavares A, Day N, Lindsay JA, Edgeworth JD, de Lencastre H, Parkhill J, Peacock SJ, Bentley SD (2010): Evolution of MRSA during hospital transmission and intercontinental spread. Science 327(5964):469-474.

Higgins PG, Dammhayn C, Hackel M, Seifert H (2010): Global spread of carbapenemresistant Acinetobacter baumannii. J Antimicrob Chemother 65:233-238.

Hill D, Rose B, Pajkos A, Robinson M, Bye P, Bell S, Elkins M, Thompson B, Macleod C, Aaron SD, Harbour C (2005): Antibiotic susceptibilities of Pseudomonas aeruginosa isolates derived from patients with cystic fibrosis under aerobic, anaerobic, and biofilm conditions. J Clin Microbiol 43:5085-5090.

Ho J, Tambyah PA, Paterson DL (2010): Multiresistant Gram-negative infections: a global perspective. Curr Opin Infect Dis 23:546-553.

Högberg LD, Heddini A, Cars $O$ (2010): The global need for effective antibiotics: challenges and recent advances. Trends in Pharmacological Sciences, 31(11), 509-515. DOI:10.1016/j.tips.2010.08.002.

IDSA (2004): public policy supplemental article. Combating antimicrobial resistance: policy recommendations to save lives. Clin Infect Dis 52:397-420. 
Jakobsson HE, Jernberg C, Andersson AF, Sjölund-Karlsson M, Jansson JK, Engstrand $L$ (2010): Short-term antibiotic treatment has differing long-term impacts on the human throat and gut microbiome. PLoS One 5:e9836.

Jarlier V, Trystram D, Brun-Buisson C, Fournier S, Carbonne A, Marty L, Andremont A, Arlet G, Buu-Hoi A, Carlet J, Decré D, Gottot S, Gutmann L, Joly-Guillou ML, Legrand P, Nicolas-Chanoine MH, Soussy CJ, Wolf M, Lucet JC, Aggoune M, Brücker G, Régnier B (2010): Curbing methicillin-resistant Staphylococcus aureus in 38 French hospitals through a 15 -year institutional control program. Arch Intern Med 170:552-559.

Jernberg C, Löfmark S, Edlund C, Jansson JK (2007): Long-term ecological impacts of antibiotic administration on the human intestinal microbiota. ISME J 1:56-66.

Katz ML, Mueller LV, Polyakov M, Weinstock SF (2006): Where have all the antibiotic patents gone? Nat Biotechnol 24:1529-1531.

Kim SK, Sims CL, Wozniak SE, Drude SH, Whitson D, Shaw RW (2009): Antibiotic resistance in bacteria: novel metalloenzyme inhibitors. Chem Biol Drug Des 74:343-348.

Köck R, Becker K, Cookson B, van Gemert-Pijnen JE, Harbarth S, Kluytmans J, Mielke M, Peters G, Skov RL, Struelens MJ, Tacconelli E, Navarro Torné A, Witte W, Friedrich AW (2010): Methicillin-resistant Staphylococcus aureus (MRSA): burden of disease and control challenges in Europe. Euro Surveill 15(41):pii=19688. http:// www.eurosurveillance.org/ViewArticle.aspx?Articleld=19688 (abgerufen am: 28. August 2012).

Kohanski MA, DePristo MA, Collins JJ (2010): Sublethal antibiotic treatment leads to multidrug resistance via radical-induced mutagenesis. Mol Cell 37:311-320.

Kumarasamy KK, Toleman MA, Walsh TR, Bagaria J, Butt F, Balakrishnan R, Chaudhary U, Doumith M, Giske CG, Irfan S, Krishnan P, Kumar AV, Maharjan S, Mushtaq S, Noorie T, Paterson DL, Pearson A, Perry C, Pike R, Rao B, Ray U, Sarma JB, Sharma M, Sheridan E, Thirunarayan MA, Turton J, Upadhyay S, Warner M, Welfare W, Livermore DM, Woodford N (2010): Emergence of a new antibiotic resistance mechanism in India, Pakistan, and the UK: a molecular, biological, and epidemiological study. Lancet Infect Dis 10:597-602.

Lee HH \& Collins JJ (2012): Microbial environments confound antibiotic efficacy. Nature Chemical Biology 8:6-9.

Li JWH \& Vederas JC (2009): Drug discovery and natural products: end of an era or an endless frontier? Science 32:161-165.

Littman DR \& Pamer EG (2011): Role of the commensal microbiota in normal and pathogenic host immune responses. Cell Host Microbe 10:311-323.

Livermore DM (2003): Bacterial resistance: origins, epidemiology, and impact. Clin Infect Dis 36 (Suppl 1):11-23.

Lujan SA, Guogas LM, Ragonese H, Matson SW, Redinbo MR (2007): Disrupting antibiotic resistance propagation by inhibiting the conjugative DNA relaxase. Proc Natl Acad Sci 104:12282-12287.

MacPherson DW, Gushulak BD, Baine WB, Bala S, Gubbins PO, Holtom P, SegarraNewnham M (2009): Population mobility, globalization and antimicrobial drug resistance. Emerg Infect Dis 15:1727-1732.

Marra A (2011): Antibacterial resistance: is there a way out of the woods? Future Microbiol 6:707-709. 
McGannon CM, Fuller CA, Weiss AA (2010): Different classes of antibiotics differentially influence shiga toxin production. Antimicrob Agents Chemother 54:3790-3798.

McGhee GC, Guasco J, Bellomo LM, Blumer-Schuette SE, Shane WW, Irish-Brown A, Sundin GW (2011): Genetic analysis of streptomycin-resistant (Sm(R)) strains of Erwinia amylovor suggests that dissemination of two genotypes is responsible for the current distribution of $\mathrm{Sm}(\mathrm{R}) \mathrm{E}$. amylovora in Michigan. Phytopathology 101(2):182-191.

Mellmann A, Harmsen D, Cummings CA, Zentz EB, Leopold SR, Rico A, Prior K, Szczepanowski R, Ji Y, Zhang W, McLaughlin SF, Henkhaus JK, Leopold B, Bielaszewska M, Prager R, Brzoska PM, Moore RL, Guenther S, Rothberg JM, Karch H (2011): Prospective genomic characterization of the German enterohemorrhagic Escherichia coli 0104:H4 outbreak by rapid next generation sequencing technology. PLoS One 6:e22751.

Mölstad S, Erntell M, Hanberger H, Melander E, Norman C, Skoog G, Lundborg CS, Söderström A, Torell E, Cars O (2008): Sustained reduction of antibiotic use and low bacterial resistance: 10-year follow-up of the Swedish Strama programme. Lancet Infect Dis 8:125-132.

Molinski TF, Dalisay DS, Lievens SL, Saludes JP (2009): Drug development from marine natural products. Nat Rev Drug Discov 8:69-85.

Mukhopadhyay J, Das K, Ismail S, Koppstein D, Jang M, Hudson B, Sarafianos S, Tuske S, Patel J, Jansen R, Irschik H, Arnold E, Ebright RH (2008): The RNA polymerase "switch region" is a target for inhibitors. Cell 135:295-307.

Nathan C, Goldberg FM (2005): Outlook: the profit problem in antibiotic R\&D. Nat Rev Drug Discov 4:887-891.

Nordmann P, Poirel L, Walsh TR, Livermore DM (2011): The emerging NDM carbapenemases. Trends Microbiol 19(12):588-595.

Nübel U, Dordel J, Kurt K, Strommenger B, Westh H, Shukla SK, Zemlicková H, Leblois R, Wirth T, Jombart T, Balloux F, Witte W (2010): A timescale for evolution, population expansion, and spatial spread of an emerging clone of methicillinresistant Staphylococcus aureus. PLoS Pathogens 6(4):e1000855.

Payne DJ, Gwynn MN, Holmes DJ, Pompliano DL (2007): Drugs for bad bugs: confronting the challenges of antibacterial discovery. Nat Rev Drug Discov 6:29-40.

Robert Koch-Institut (2011): Epidemiologisches Bulletin, 13/2011.

Robert Koch-Institut (2012): Epidemiologisches Bulletin, 11/2012.

Rybak MJ (2006): Pharmacodynamics: relation to antimicrobial resistance. Am J Infect Control 34 (5 Suppl 1): 38-45; discussion 64-73.

Sabuncu E, David J, Bernède-Bauduin C, Pépin S, Leroy M, Boëlle PY, Watier L, Guillemot D (2009): Significant reduction of antibiotic use in the community after a nationwide campaign in France, 2002-2007. PLoS Med 2009 Jun 2;6(6):e1000084. Epub 2009 Jun 2.

Schwabe U \& Paffrath D (Hrsg.) (2011): Arzneiverordnungs-Report 2011. SpringerVerlag, Berlin, Heidelberg, New York.

Silver LL (2011): Challenges of Antibacterial Discovery. Clinical Microbiology Reviews 24(1):71-109.

Silver LL (2011b): Scientific Obstacles to Discovery of Novel Antibacterials. Background document commissioned by ReAct for the seminar "Collaboration for innovation The Urgent Need for New Antibiotics”, Brüssel, 23. Mai 2011. 
So AD, Gupta N, Brahmachari SK, Chopra I, Munos B, Nathan C, Outterson K, Paccaud JP, Payne DJ, Peeling RW, Spigelman M, Weigelt J (2011): Towards new business models for R\&D for novel antibiotics. Drug Resistance Updates 14:88-94.

Sriramulu DD, Lünsdorf H, Lam JS, Römling U (2005): Microcolony formation: a novel biofilm model of Pseudomonas aeruginosa for the cystic fibrosis lung. J Med Microbiol 54:667-676.

Stecher B, Denzler R, Maier L, Bernet F, Sanders MJ, Pickard DJ, Barthel M, Westendorf AM, Krogfelt KA, Walker AW, Ackermann M, Dobrindt U, Thomson NR, Hardt WD (2012): Gut inflammation can boost horizontal gene transfer between pathogenic and commensal Enterobacteriaceae. Proc Natl Acad Sci 109:1269-1274.

Stecher B \& Hardt WD (2011): Mechanisms controlling pathogen colonization of the gut. Curr Opin Microbiol 14:82-91.

Trautmann M, Scheibe C, Wellinghausen N, Holst O, Lepper PM (2010): Low endotoxin release from Escherichia coli and Bacteroides fragilis during exposure to moxifloxacin. Chemotherapy 56:364-370.

Turnidge J \& Paterson DL (2007): Setting and revising antibacterial susceptibility breakpoints. Clin Microbiol Rev 20:391-408.

van de Sande-Bruinsma N, Grundmann H, Verloo D, Tiemersma E, Monen J, Goossens $\mathrm{H}$, Ferech M (2008): Antimicrobial drug use and resistance in Europe; European Antimicrobial Resistance Surveillance System Group; European Surveillance of Antimicrobial Consumption Project Group. Emerg Infect Dis 14:1722-1730.

van Loo I, Huijsdens X, Tiemersma E, de Neeling A, Sande-Bruinsma N van de, Beaujean D, Voss A, Kluytmans J (2007): Emergence of methicillin-resistant Staphylococcus aureus of animal origin in humans. Emerg Infect Dis 13(12):1834-1839.

Vianna RC, Gomes RN, Bozza FA, Amâncio RT, Bozza PT, David CM, Castro-Faria-Neto HC (2004): Antibiotic treatment in a murine model of sepsis: impact on cytokines and endotoxin release. Shock 21:115-120.

Voss A, Loeffen F, Bakker J, Klaassen C, Wulf M (2005): Methicillin-resistant Staphylococcus aureus in pig farming. Emerg Infect Dis 11(12):1965-1966.

Weighardt H \& Holzmann B (2007): Role of Toll-like receptor responses for sepsis pathogenesis. Immunobiology 212:715-722.

Wenzel SC \& Müller R (2009): The biosynthetic potential of myxobacteria and their impact on drug discovery. Current Opinion in Drug Discovery \& Development 12(2):220-230.

White AR (2011): Effective antibacterials: at what cost? The economics of antibacterial resistance and its control. Journal of Antimicrobial Chemotherapy. DOI:10.1019/ $\mathrm{jac} / \mathrm{dkr} 260$.

WHO (2011): WHO Report 2011 Global tuberculosis control. http://whqlibdoc.who.int/ publications/2011/9789241564380_eng.pdf.

Wilke MH (2010): Multiresistant bacteria and current therapy - the economical side of the story. Eur J Med Res 15:571-576.

Witte W \& Cuny C (2011): Emergence and spread of cfr-mediated multiresistance in staphylococci: an interdisciplinary challenge. Future Microbiol 6:925-931.

Wulf M \& Voss A (2008): MRSA in livestock animals - an epidemic waiting to happen? Clin Microbiol Infect 14(6):519-521.

Zilberberg MD \& Shorr AF (2010): Understanding cost-effectiveness. Clinical Microbiology and Infection 16:1707-1712. 